\title{
TRANSPLANTE HEPÁTICO, ADOLESCÊNCIA E TRANSIÇÃO PARA AMBULATÓRIO ADULTO
}

\author{
Liver transplantation, adolescence and transition to adults' clinic
}

\author{
Beatriz Elizabeth Bagatin Veleda Bermudez', Lídia Natália Dobrianskyj Weber², Mônica Beatriz Parolin, Júlio Cezar Uilli Coelho4, \\ Mariane Bagatin Bermudez ${ }^{5}$, Adriane Celli ${ }^{6}$
}

\begin{abstract}
RESUMO
Objetivo: Fornecer informações ao hepatologista, referentes ao atendimento ao adolescente transplantado, no que diz respeito às questões da adolescência para evitar risco de rejeição, muito prevalente nesse grupo. As informações foram coletadas de artigos científicos publicados nas bases de dados SciELO, MEDLINE e PUBMED de 2004 a 2010, livros técnicos e publicações de consenso de organismos internacionais. Síntese dos dados: Com o aumento do número de transplantes e melhora da sobrevida, muitas crianças transplantadas estão atingindo a fase turbulenta da adolescência e muitos adolescentes são submetidos ao transplante nessa fase. Na atualidade, poucos serviços de transplante têm padronizado um programa de transição, para assegurar ao paciente adolescente um seguimento de saúde adequado após sua transferência para uma clínica de adultos. Alguns autores confirmam que a atenção no período de transição possibilita uma revisão educacional para os pacientes adolescentes e também uma oportunidade conveniente para otimizar o bem-estar na vida adulta. Infelizmente, porém, as clínicas de adultos em geral estão sobrecarregadas de pacientes e as questões do desenvolvimento da adolescência não são prioritárias. O pediatra, que deveria ter um papel essencial na transferência de adolescentes para serviços de saúde de adultos, muitas vezes e de modo inconsciente, acaba atuando como um dificultador por não demonstrar confiança plena em seus colegas. Conclusão: Um ambulatório de transição oferece a oportunidade de abordar questões essenciais para a saúde integral do adolescente transplantado e seu desenvolvimento pleno com temas como: autonomia, identidade, auto-estima, qualidade de vida, sexualidade, alterações corporais, projetos de vida e desenvolvimento da escolaridade/profissionalização.
\end{abstract}

Descritores: Adolescência, Transplante Hepático, Adesão à Medicação.

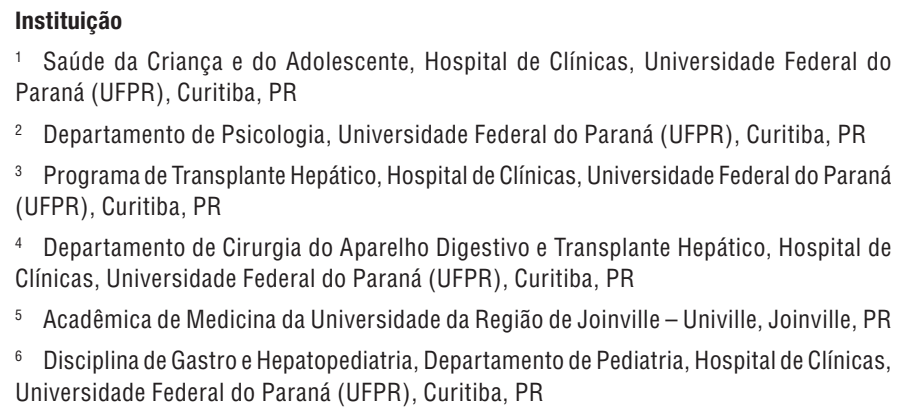

4 Departamento de Cirurgia do Aparelho Digestivo e Transplante Hepático, Hospital de Clínicas, Universidade Federal do Paraná (UFPR), Curitiba, PR

5 Acadêmica de Medicina da Universidade da Região de Joinville - Univille, Joinville, PR

6 Disciplina de Gastro e Hepatopediatria, Departamento de Pediatria, Hospital de Clínicas, Universidade Federal do Paraná (UFPR), Curitiba, PR

\section{Correspondência:}

Beatriz Elizabeth Bagatin Veleda Bermudez

Rua Desembargador Westphalen, 15 - Cj.1405, CEP 80010-110 - Curitiba/PR

Fone: (41) 3224-6476

E-mail: beatriz_bvb@hotmail.com

\section{INTRODUÇÃO}

O transplante hepático é o tratamento de eleição para doenças avançadas do fígado e é o transplante de órgão sólido mais bem sucedido, com sobrevida de um ano de 83 a $91 \%$ e de cinco anos de 82 a $84 \%$, dependendo da idade do receptor. ${ }^{1}$ As principais indicações para transplante hepático em crianças e adolescentes são as doenças hepáticas colestáticas $(48 \%)$, seguidas de insuficiência hepática fulminante $(11 \%)$, doenças hepáticas metabólicas (13\%), tumores hepáticos $(4 \%)$ e outras doenças (9\%). ${ }^{1}$ Entre os objetivos dos centros de transplante hepático estão o manejo e a melhora na transferência dos adolescentes aos serviços dos adultos. ${ }^{2}$

A adolescência é uma etapa crucial e bem definida do processo de crescimento e desenvolvimento, cuja marca registrada é a transformação ligada aos aspectos físicos e psíquicos do ser humano, com intenso desenvolvimento cerebral até a terceira década de vida. ${ }^{3-5}$ Esse período é extremamente relevante para a construção do sujeito individual e social, devendo, porém ser considerados a vulnerabilidade e o risco do uso indevido de drogas, 
DST, AIDS e gravidez não planejada, além de apresentar pico de incidência para doença mental.

Paralelamente às modificações corporais (crescimento físico, eclosão hormonal, maturação sexual, que se denomina puberdade), evoluem aquelas de ordem psicossocial, ${ }^{6}$ no que convencionaram chamar de síndrome da adolescência normal, que são: busca da identidade, tendência grupal, desenvolvimento do pensamento abstrato, vivência temporal singular, variações do humor, evolução da sexualidade, separação progressiva dos pais, crises religiosas, atitude social reivindicatória e manifestações contraditórias de conduta. A necessidade de manter um compromisso com um acompanhamento médico contínuo é um fator adicional para o estresse próprio dessa fase da vida. ${ }^{7}$

\section{ADOLESCÊNCIA E TRANSPLANTE}

Em 2008, representantes de vários grupos de transplante hepático reuniram-se para discutir sobre a transição de seus pacientes adolescentes para o ambulatório de adultos, a fim de garantir a continuidade do sucesso em longo prazo, apesar da dependência da medicação imunossupressora. Houve concordância sobre a necessidade de treinamento e estudos na área da adolescência de pesquisas sobre as melhores práticas e análise da evolução, bem como sobre a utilização dos recursos da comunidade. ${ }^{8}$

A adolescência é um período turbulento para todos, e, particularmente, para esses pacientes. Adolescentes com doenças crônicas apresentam mais problemas de ajustamento que seus pares sem doenças e tendem a sofrer mais transtornos de comportamento, depressão e ansiedade. ${ }^{9}$ Para diminuir o impacto que uma doença crônica presente no período da infância causa no curso da vida, eles devem ser estimulados a ter autonomia e manter contato social com seus pares, mesmo necessitando de cuidados da família e dos profissionais de saúde. ${ }^{9}$

Adolescentes com transplante de órgão sólido e idade entre 18 e 24 anos apresentam o dobro da taxa de mortalidade com relação àqueles entre 12 e 17 anos e quatro vezes maior aos de 10 a 14 anos. O uso de drogas também aumenta e a taxa de suicídio é três vezes maior que a de adolescentes entre 12 e 17 anos..$^{5}$ Adultos jovens e adolescentes transplantados renais têm as taxas mais altas de rejeição aguda, morte por perda do enxerto e rejeição crônica, levando à perda do enxerto. ${ }^{8}$ Mais do que um terço dos adolescentes não aderem ao tratamento. ${ }^{8}$ A tendência normal ao questionamento e desafio à autoridade pode predispor esses pacientes a aceitar com desdém as orientações médicas e o tratamento. Além disso, devem ser considerados os efeitos cosméticos adversos da terapia imunossupressora e a preocupação com a imagem corporal, a impulsividade e o comportamento de risco. ${ }^{8}$ Segundo Bell, imagens estruturais e funcionais do cérebro mostram que alterações significativas na maturação ainda ocorrem aos 20 anos e essas regiões que demoram a amadurecer estão associadas às funções executivas cerebrais, tais como previsão, planejamento, avaliação dos riscos e capacidade para dissociar a tomada de decisão da emoção forte.

Embora em alguns trabalhos, adolescentes com doenças crônicas pareçam apresentar maior risco, Dommergues ${ }^{10} \mathrm{em}$ seu estudo com 116 pacientes (abril/05 a julho/06 e idade 21+/-4 anos) observou que o comportamento de risco não apresentou diferença em relação a seus pares normais referente ao uso de tabaco (11\%) e maconha (11\%), mas encontrou taxas significativamente menores nesses pacientes quanto ao uso de álcool (43\% nunca usaram e $50 \%$ bebem eventualmente em ocasiões especiais).

\section{ADERÊNCIA NA ADOLESCÊNCIA}

A sobrevida após um ano de transplante de órgão sólido é melhor em receptores adolescentes (idade 11-19 anos) do que nos mais jovens. No entanto, muitos estudos indicam piora nos seguimentos em longo prazo (mais de cinco anos pós-transplante) no grupo adolescente do que em crianças de menor idade, fato explicado pela não aderência. ${ }^{11} \mathrm{O}$ adolescente vai desenvolvendo a separação de sua identidade da dos pais, e essa necessidade de independência combinada com a dificuldade de entender conceitos abstratos e a noção de invulnerabilidade pode resultar em pensamentos ilógicos que poderão determinar um comportamento de risco. ${ }^{7}$

Todos os receptores de transplante, inclusive os adolescentes, utilizam imunossupressores por toda vida, porém a prevalência geral de não aderência é 32\% para transplante renal e 30,8\% para o de fígado. Ao fazer a divisão por idade, ela é de $38 \%{ }^{12}$ a $64 \%{ }^{11} \mathrm{em}$ adolescentes, $17 \%$ em crianças e $15-25 \%$ em adultos. ${ }^{13,11}$ Para Hsu ${ }^{7}$ é de $7-70 \%$, com níveis mais altos na adolescência.

A qualidade da comunicação entre médicos e adolescentes pode comprometer o entendimento de sua condição de saúde, a satisfação com o tratamento, a colaboração e o compromisso com os cuidados de saúde. ${ }^{5}$

Pela OMS, há cinco categorias de fatores de risco para a não aderência ao tratamento, conforme Figura 1.

A aderência da população pediátrica e de adolescentes pode aumentar em 56\% quando estratégias educativas são tentadas continuadamente. Entre elas: a) explicação do nome e objetivo de cada medicamento, dose e horário, seus efeitos colaterais; b) informar sobre sinais de rejeição ou infecção, com envolvimento do adolescente como um parceiro; c) conhecer experiências prévias da doença do paciente, seu entendimento e suas crenças; d) tentar não ser autoritário ao prover a orientação a ser seguida não só verbalmente, mas também por escrito, e fornecer panfletos, vídeos ou livretos. ${ }^{11} \mathrm{O}$ paciente deve ser inquirido para conferir se houve boa compreensão. ${ }^{11}$ Como a aderência decresce ao longo do tempo, é importante repetir sistematicamente as orientações. Entre as estratégias comportamentais, deve existir um momento para que os adolescentes possam ser ouvidos a cada consulta sobre suas necessidades, sentimentos ou dificuldades com a medicação. Para facilitar seu uso correto, a medicação pode ser simplificada em número de doses, no sabor ou tamanho. Lembretes no calendário ou agenda, folhetos, alarme de relógio ou telefone celular, ou ainda a associação com alguma atividade também melhoram a aderência. ${ }^{11}$ É importante que os pais mantenham um diálogo sincero e honesto com a equipe médica e que haja supervisão contínua sobre o adolescente, até que ele desenvolva sua responsabilidade e autonomia, sempre respeitando sua individualidade. Do mesmo modo, a equipe de saúde deve manter respeito e confiança, valorizando o paciente integralmente como pessoa, facilitando o acesso e a comunicação aberta, quer seja pessoalmente, por telefone ou por e-mail, para que ocorra fortalecimento do vínculo com os profissionais de saúde. ${ }^{11}$ Caso exista conflito pessoal ou familiar, deve-se prover terapia familiar, tratamento psicológico individual ou em grupo e encaminhamento psiquiátrico no caso de 
Figura 1: Fatores de risco à aderência dos adolescentes transplantados de fígado

\begin{tabular}{|c|c|c|c|}
\hline Fatores socioeconômicos & $\begin{array}{l}\text { Fatores relacionados ao } \\
\text { paciente }\end{array}$ & Fatores relacionados à doença & $\begin{array}{l}\text { Fatores relacionados ao serviço } \\
\text { de saúde }\end{array}$ \\
\hline $\begin{array}{l}\text { - Cultura e raça } \\
\text { - Baixa condição econômica } \\
\text { - Custo da medicação } \\
\text { - Instabilidade familiar } \\
\text { - Família desequilibrada } \\
\text { - Pouca coesão familiar } \\
\text { - Falta de supervisão parental } \\
\text { - Comunicação insuficiente entre } \\
\text { pais e filhos } \\
\text { - Ansiedade e superproteção do } \\
\text { paciente pelos pais } \\
\text { - Família monoparental } \\
\text { - Isolamento social } \\
\text { - Não pertencer a um grupo/ } \\
\text { isolamento social }\end{array}$ & $\begin{array}{l}\text { - Pouco entendimento da } \\
\text { doença } \\
\text { - Baixa autoestima } \\
\text { - Imagem corporal ruim } \\
\text { - Esquecimento } \\
\text { - Estilo de vida agitado } \\
\text { - Esquema de vida muito } \\
\text { ocupado } \\
\text { - Deficiência mental } \\
\text { - Não aderência prévia } \\
\text { - Depressão ou distresse } \\
\text { psicológico } \\
\text { - Estresse pós-traumático } \\
\text { - Raiva } \\
\text { - Evasão escolar } \\
\text { - Comportamento de risco } \\
\text { - História de abuso na infância } \\
\text { - Mecanismo de enfrentamento } \\
\text { pobre } \\
\text { - Negação } \\
\text { - Problemas de ajustamento } \\
\text { social } \\
\text { - Poucas habilidades sociais } \\
\text { - Luta pela autonomia }\end{array}$ & $\begin{array}{l}\text { - Duração da doença } \\
\text { - Percepção da vulnerabilidade: } \\
\text { assintomático e boa saúde } \\
\text { - Muito tempo pós-transplante } \\
\text { - Doador de órgão vivo } \\
\text { - Abuso de droga } \\
\text { Fatores relacionados } \\
\text { ao tratamento } \\
\text { - Efeitos adversos cosméticos } \\
\text { - Número total de medicamentos } \\
\text { - Número de doses diárias } \\
\text { - Cronicidade e complexidade do } \\
\text { esquema terapêutico } \\
\text { - Gosto da medicação } \\
\text { - Tamanho dos comprimidos } \\
\text { - Custo da medicação }\end{array}$ & $\begin{array}{l}\text { - Pouca comunicação entre o } \\
\text { médico responsável, paciente e } \\
\text { família } \\
\text { - Estilo de comunicação } \\
\text { autoritário da equipe } \\
\text { - Habilidade didática pobre da } \\
\text { equipe } \\
\text { - Pouco conhecimento da não } \\
\text { aderência à medicação } \\
\text { - Interferência da evolução na } \\
\text { escola ou trabalho } \\
\text { - Raiva ou perda de esperança } \\
\text { da família na equipe de saúde } \\
\text { - Perda da continuidade do } \\
\text { acompanhamento } \\
\text { - Provedor de saúde único } \\
\text { - Intervalo longo entre as } \\
\text { consultas } \\
\text { - Perda de acompanhamento } \\
\text { - Problema de acesso aos } \\
\text { cuidados }\end{array}$ \\
\hline
\end{tabular}

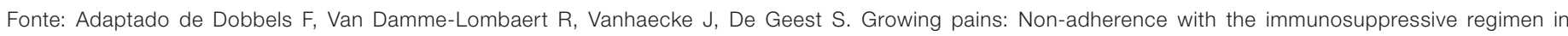
adolescent transplant recipients. Pediatr Transplantation 2005: 9: 381-390.

transtorno mental ou comportamental. ${ }^{11}$

A não aderência é um processo dinâmico: um paciente pode apresentar-se não aderente em um momento e aderente em outro, pois não há um método que meça continuamente o nível das drogas imunossupressoras. ${ }^{14}$ Parece que os jovens usam a medicação mais adequadamente quando acreditam que seu provedor de saúde realmente se interessa por eles e explica as razões para seu uso correto, assim como quando permite sua participação nas decisões, proporcionando alterações que se ajustem ao seu estilo de vida, com esquemas úteis e práticos. ${ }^{14}$

\section{CRESCIMENTO E DESENVOLVIMENTO}

Quando crianças com déficit pôndero-estatural são transplantadas precocemente, a recuperação da estatura potencial é frequente, enquanto que o transplante tardio dificulta atingir esse potencial. ${ }^{15}$ Essa rapidez de modulação provavelmente é devida ao fator 1 de crescimento insulinalike e sugere que essa substância seja um indicador precoce de recuperação da função hepática. ${ }^{16}$ Os meninos crescem mais do que as meninas, não havendo correlação com a imunossupressão e com o tipo do transplante. O crescimento estatural insuficiente (na faixa de -1 a 1,5 no escore $\mathrm{z}$ ) prévio ao transplante pode resultar em impacto negativo no crescimento potencial e no desenvolvimento cognitivo pós-transplante. Isso ocorre devido às alterações metabólicas da desnutrição grave (má absorção de gorduras, metabolismo de nitrogênio anormal e aumento do gasto energético), bem como pela resistência ao hormônio do crescimento $(\mathrm{GH}) .{ }^{17}$ Esse hormônio pode até estar elevado nessas crianças e ocorre quando há falha na produção do fator de crescimento insulina-like (IGFI) produzido no fígado e da proteína 3 que se liga ao IGFI. ${ }^{17}$ Embora haja uma recuperação evidente em muitas crianças após o transplante, muitas não superam esse déficit. ${ }^{17}$ A exposição ao corticóide é outro fator que interfere no crescimento e a maioria dos protocolos diminui ou suspende essa droga entre seis e 18 meses pós-transplante. A administração de $\mathrm{GH}$ recombinante melhora a velocidade de crescimento em dois a três anos de uso sem aumento da idade óssea, porém, pode contribuir para o risco de rejeição tardia do enxerto. ${ }^{17}$

Foram avaliados 432 pacientes por 24 anos pós-transplante hepático com função hepática normal e foi evidenciado que o ritmo de crescimento é acelerado, com recuperação de peso e altura ao final do primeiro ano, principalmente em crianças menores de dois anos. ${ }^{17}$ No entanto, nem sempre há recuperação total, sendo as principais variáveis: diabetes, corticóide, doença hepática primária, família monoparental e transplante feito antes de 2001. ${ }^{17}$

A função cognitiva é normal na maioria das crianças transplantadas, mas algumas apresentam distúrbio de aprendizagem (17,7-26\% versus $8 \%$ na população normal). Quando isso ocorre, suas causas devem ser exploradas; entre elas, o déficit auditivo, que 
pode ocorrer em 5\% das crianças transplantadas. ${ }^{17}$ Pacientes com distúrbio de aprendizagem necessitam de suporte educacional individualizado. ${ }^{17}$ Pais de crianças transplantadas de fígado relatam mais estresse e tendem a avaliar seus filhos com desempenho mais baixo do que o relatado por elas próprias. ${ }^{17}$

Importante lembrar que devem ser aplicadas todas as vacinas possíveis antes do transplante, pois após o procedimento, devido à imunossupressão, estarão contra-indicadas as vacinas de vírus vivo. ${ }^{18}$

\section{SAÚDE SEXUAL E REPRODUTIVA}

A melhora da sobrevida dos receptores de órgão sólido fez aumentar a atenção para a qualidade de vida desse grupo, que inclui preocupações com o crescimento, envolvimento amoroso e desejo de controlar a fertilidade. ${ }^{19}$

A complexidade dos cuidados com adolescentes receptoras de órgãos sólidos exige muito empenho dos profissionais de saúde..$^{19} \mathrm{~A}$ puberdade é comumente atrasada em um a dois anos com menarca variando entre 13 e 19 anos, quando o transplante hepático ocorreu em torno dos 10 anos. ${ }^{1}$ Esse atraso poderá ocorrer em meninas receptoras de rim ou fígado no período pré-puberal e pode criar dificuldades na discussão dessas questões com a equipe de saúde ${ }^{19}$.

Um motivo de preocupação nos meninos é a ginecomastia, que pode ser causada por drogas como digoxina, isoniazida, espironolactona e inclusive a maconha; eles devem ser tranquilizados quanto a esse comum efeito colateral e informados quanto ao fato de que irá desaparecer. É importante salientar que não se trata de mudança de sexo e que eles não devem comprimir o tecido mamário, a fim de não estimular seu crescimento. ${ }^{14}$

Meninos e meninas podem ter faltado às aulas de educação sexual na escola e a disponibilidade de material educativo (folders, panfletos, revistas, vídeos, etc.) sobre adolescência pode facilitar discussão sobre essas questões nas consultas. ${ }^{14}$ Deve-se focar imagem corporal positiva, assertividade, informações sobre anatomia, funcionamento sexual, espectro de possibilidades sexuais, papéis sexuais, habilidades sociais, contracepção e prevenção de doenças sexualmente transmissíveis ${ }^{14}$. Crenças, conhecimento e comportamento responsável são influenciados pela cultura, classe social, habilidade cognitiva, sexo, orientação sexual e estado geral de saúde. ${ }^{14}$

A maioria dos estudos sugere que transtornos menstruais prévios ao transplante e relacionados à disfunção hepática terminam em média sete meses após o transplante. ${ }^{19} \mathrm{O}$ tratamento do sangramento uterino disfuncional é feito com contraceptivo hormonal. Essas drogas, assim como suplementos nutricionais e fitoterápicos prescritos pelas próprias adolescentes podem interagir com o regime imunossupressor usado. ${ }^{19}$ Deve-se orientar a evitar o uso de tais substâncias sem antes consultar seu médico.

O envolvimento amoroso de adolescentes receptoras de órgão sólido é limitado pelo isolamento social por elas vivenciado em relação a seus pares normais. ${ }^{19}$ A cicatriz cirúrgica na infância pode comprometer a imagem corporal, com risco de isolamento devido ao medo de rejeição de potenciais parceiros sexuais. ${ }^{20}$ Em adultos transplantados é alta a incidência de disfunção sexual. No entanto, não parece existir maior incidência dessa disfunção detectada em entrevistas com adolescentes sexualmente ativos submetidos a transplante, quando comparados à população em geral. ${ }^{19}$

Nem a medicação imunossupressora nem o transplante diminuem a fertilidade e já se descreveu a concepção três semanas após o transplante; porém, a recomendação é que esta ocorra pelo menos seis a doze meses após. ${ }^{19}$ Semelhante a seus pares saudáveis, as adolescentes têm dificuldades de conversar com profissionais de saúde sobre a necessidade de contracepção. Isso provavelmente ocorre porque muitos pais insistem em acompanhálas em todos os momentos de sua consulta e também porque os médicos subestimam sua vida sexual ativa e sua necessidade de contracepção, possibilitando a gravidez não planejada. ${ }^{19}$ Mulheres sexualmente ativas podem escolher contraceptivos combinados de estrogênio e progesterona, só progesterona, métodos de barreira como preservativo, diafragma, DIU, capuz cervical e contracepção de emergência, se ocorreu episódio de sexo não protegido, além de sua esterilização cirúrgica ou do parceiro na idade adulta. ${ }^{19}$

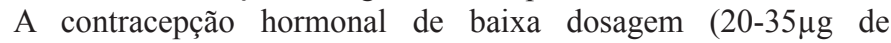
estrógeno) não deve ser prescrita para pacientes com história de tromboembolismo, hipertensão, choque ou doença coronariana, câncer sensível a estrogênio, adenoma hepatocelular e doença hepática ativa. ${ }^{19} \mathrm{O}$ método de barreira mais comumente associado é o preservativo masculino, prevenindo gravidez e infecções. ${ }^{19}$

As interações dos imunossupressores com contraceptivos hormonais estão na Figura 2. ${ }^{19}$ Autores já mostraram vantagens e desvantagens sobre contraceptivos nessa mesma pesquisa ${ }^{19}$.

Um ano após o transplante, 97\% das mulheres restabeleceram seu metabolismo estrogênico. ${ }^{21} \mathrm{O}$ aumento da sobrevida póstransplante hepático contribuiu para aumento no número de gestações com risco de gravidez ectópica, hipertensão arterial, eclâmpsia, infecção, prematuridade, retardo de crescimento intrauterino e malformações, sem associação com o tipo de terapia imunossupressora, que não pode ser descontinuada na gravidez. ${ }^{21}$

Dentre 143 partos hospitalares com predomínio de cesárea, foi observada mortalidade materna semelhante entre casos e controles, porém pacientes transplantadas apresentaram taxas mais elevadas de mortalidade fetal e complicações fetais, com mais internações pré-parto e por causa materna. ${ }^{22}$ Hipertensão na gravidez e hemorragia pós-parto foram mais frequentes entre pacientes transplantadas, e seus filhos tiveram maiores taxas de prematuridade, distresse respiratório e restrição de crescimento, mas não anomalias congênitas. ${ }^{22}$

Infecção é uma complicação e uma causa significativa de mortalidade e morbidade no receptor de transplante de órgão sólido e por isso é importante conseguir uma história sigilosa, e, se houver atividade sexual, o adolescente deve ser referenciado à profissional que cuida de saúde sexual e reprodutiva, mesmo que confidencialmente. ${ }^{19}$ Deve ser solicitado screening a cada seis meses para blenorragia e clamídia, as duas bactérias mais comuns em adolescentes ou se houver leucorreia, disúria ou outros sintomas genitourinários. ${ }^{19}$ Disúria pode ser o único sintoma de infecção por clamídia. ${ }^{22} \mathrm{Na}$ presença de sintomas, deve ser iniciado tratamento empírico para DST, para evitar doença inflamatória pélvica, que pode ser complicada com abscesso tubo-ovariano, presente às vezes em mulheres imunossuprimidas com sintomas leves devido a bactérias incomuns nessa localização como Pseudomonas aeruginosa e Aspergillus. ${ }^{19}$ Também infecções virais podem ocorrer em adolescentes, incluindo hepatite B, herpes simples e HPV, sendo a vacinação contra hepatite B e HPV da maior importância. ${ }^{19} \mathrm{O}$ exame de Papanicolau deve ser feito anualmente em adolescentes sexualmente ativas. ${ }^{19}$ 
Figura 2 - Efeitos colaterais dos imunossupressores e interações com contraceptivos orais (COC)

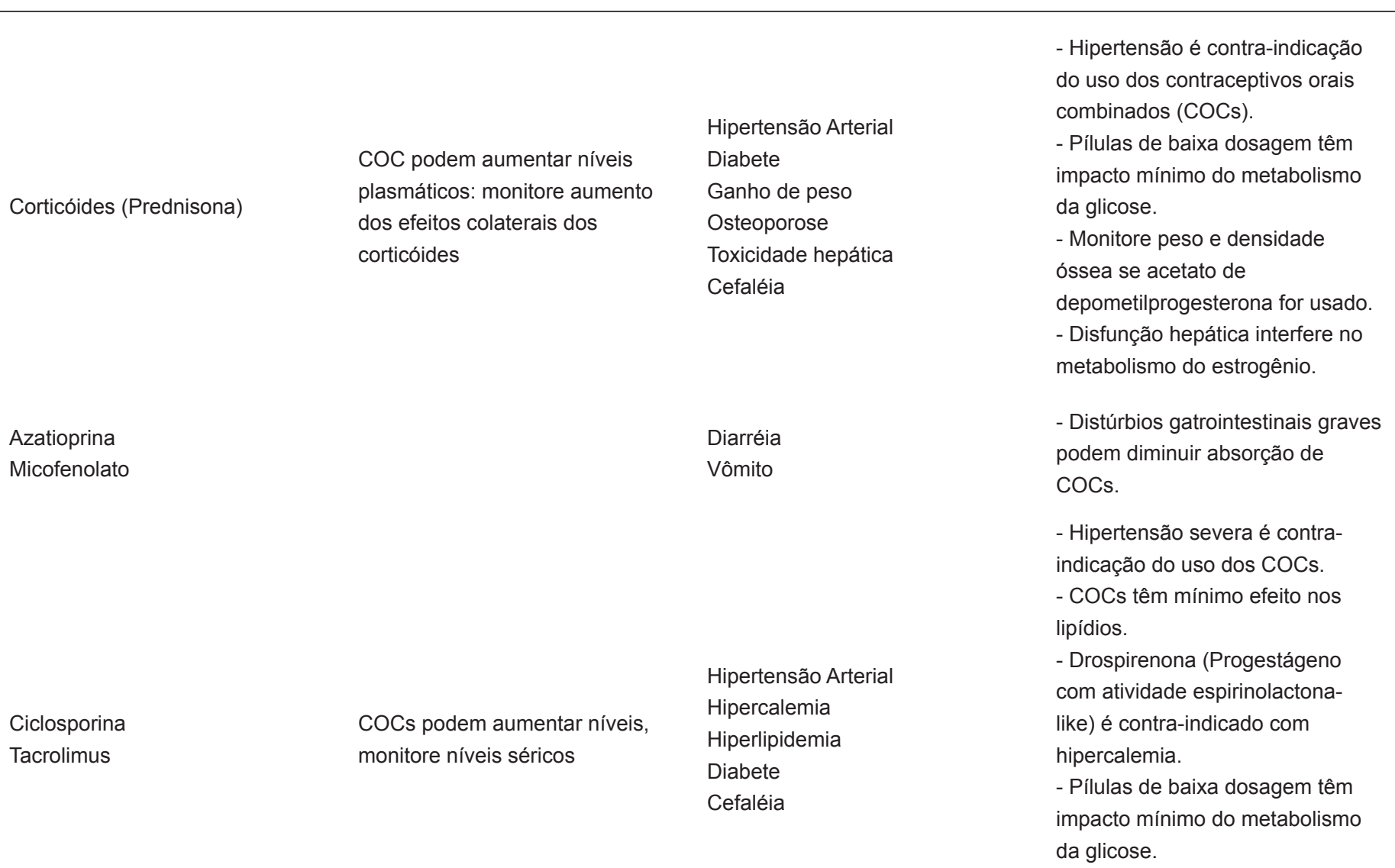

-Monitore cefaléia com freqüência.

Sirolimus

COCs podem aum

entar níveis, monitore níveis séricos.
Hiperlipidemia
- COCs têm mínimo efeito nos lipídios.

Fonte: Sucato GS, Murray PJ. Gynecologic health care for adolescent solid organ transplant recipient. Pediatr Transplant. 2005 Jun;9(3):350

\section{SAÚDE MENTAL}

A prevalência de depressão entre adolescentes da comunidade é de 5 a $14 \%$, aumentando até a vida adulta, e mais de $60 \%$ dos adolescentes deprimidos apresentam transtorno de ansiedade, mostrando alta incidência de problemas emocionais entre adolescentes submetidos a transplante de órgão sólido. ${ }^{14}$ Entre os fatores pré-transplante correlacionados com transtornos psiquiátricos após o procedimento estão: história psiquiátrica pregressa, sexo feminino e baixo suporte familiar ${ }^{14}$. Ansiedade ou depressão podem interferir na adesão ao tratamento e esses pacientes devem ser prontamente encaminhados à avaliação psiquiátrica. ${ }^{14}$

O aparecimento de transtornos alimentares (anorexia nervosa, bulimia nervosa, entre outros) é mais comum na adolescência com comorbidade com outros transtornos psiquiátricos e, por serem difíceis de tratar, têm alta morbidade e mortalidade. Os fatores de risco são: gênero feminino, problemas alimentares anteriores, desnutrição na infância e peso materno. Os fatores de proteção para esses transtornos são alta auto-estima aos 10 anos e bem-estar materno. ${ }^{14}$ Apesar de não estar clara a causa de transtorno alimentar pós-transplante, a experiência clínica defende discutir imagem corporal, seguindo o ato de pesar e reforçar uma dieta saudável. ${ }^{14}$

Psicoterapia cognitivo-comportamental, intervenções terapêuticas de suporte, terapia de grupo e terapia familiar podem auxiliar o adolescente a identificar e expressar seus sentimentos, aceitar sua condição de saúde, bem como seu tratamento. ${ }^{14}$ Dentre os medicamentos mais usados estão os inibidores seletivos de recaptação da serotonina, sendo a fluoxetina a mais indicada para depressão. O citalopram e o escitalopram tendem a ter interações menores e menos efeitos colaterais. ${ }^{14}$

Muitos programas de transplante hepático instituem tolerância zero para uso de qualquer droga lícita ou ilícita, pois além dos seus malefícios, há o risco de esquecer de tomar a medicação prescrita. ${ }^{14}$ Os adolescentes transplantados devem ser aconselhados a não 
fumar, pois tabagistas têm mortalidade maior pós-transplante, decorrente de doença cardiovascular e sepse. ${ }^{23} \mathrm{Um}$ terço dos receptores de fígado parou de fumar após o transplante e $12 \%$ de todos os transplantados são tabagistas ativos. Foi constatado que após 10 anos do transplante houve $12,7 \%$ de doenças malignas (incluindo pele) nos tabagistas contra 2,1\% nos não tabagistas. ${ }^{24}$

O abuso de álcool e drogas ilícitas após o transplante pode levar a não aderência ao tratamento imunossupressor, de acordo com uma meta-análise e os fatores de risco para recaída são: suporte social deficiente, história de álcool na família e período de abstinência menor do que seis meses. ${ }^{25}$

\section{QUALIDADE DE VIDA}

O conceito de qualidade de vida é multidimensional: bem estar físico, mental e social. ${ }^{26}$ Os fatores fisiológicos são os indicadores clínicos de saúde, nível de energia e status funcional, que incluem tolerância à dor e percepção individual. ${ }^{26}$ Os fatores psicológicos englobam nível emocional, auto-estima, imagem corporal e aspectos cognitivos. ${ }^{26}$ Os fatores sociais incluem saúde social (habilidade para desempenhar seu papel social por si só) e suporte social, que modera a percepção de eventos estressantes. ${ }^{26}$

Numa avaliação sobre qualidade de vida em 123 pacientes antes e após transplante hepático, constatou-se que era considerada boa pela maioria $(79 \%)$, muito boa $(14 \%)$ e má $(5 \%){ }^{27}$

Adolescentes que receberam transplante hepático têm qualidade de vida relacionada à saúde similar a adolescentes normais em avaliação pessoal. No entanto, os pais desses pacientes consideramna diminuída e todos os estudos nesse sentido evidenciam a presença de distresse emocional parental e atividades familiares disruptivas. ${ }^{17}$ Em crianças menores de cinco anos foi observado que houve melhora da qualidade de vida quando esse escore foi comparado entre a fase pré-transplante e cinco anos depois. ${ }^{28}$

Um estudo finlandês realizado com 353 transplantados de fígado com idade variando de menos de 20 até mais de 50 anos constatou que a qualidade de vida é melhor em transplantados que trabalham na vida adulta do que naqueles que vivem dos benefícios do governo. ${ }^{29}$

Figura 3 - Marcos da transição de adolescentes para o ambulatório de adultos
Ao observarem 38 pacientes com idade variando de 28 meses a 16 anos, Fredericks e cols. ${ }^{30}$ concluíram que, para melhorar a qualidade de vida, é muito importante identificar e fazer intervenções clínicas em transplantados que apresentam baixo desempenho físico, maior limitação da atividade social e escolar relacionada a problemas emocionais e comportamentais, bem como naqueles com pais com estresse e com baixa coesão familiar. ${ }^{31}$

Um estudo anterior observou 300 adolescentes transplantados de órgão sólido quanto às suas experiências e seus sentimentos e concluíram que existe percepção de dominação pelos esquemas terapêuticos, auto-estima diminuída, ressentimento de sentir emoções de modo diferente, reação negativa de seus pares, perda do sentimento de pertencer, ansiedade de ser rejeitado, duração do transplante e incerteza sobre sua expectativa de vida. ${ }^{32}$ Alguns tinham ideações suicidas, outros estavam agradecidos pelo transplante ter dado nova chance de vida e outros ainda desejavam mais independência e se sentiam superprotegidos pelos pais, sendo que o desejo de definir uma meta acadêmica e profissional de longo prazo foi prevalente. ${ }^{32}$

Um estudo com 395 crianças e adolescentes transplantados hepáticos entre 1981 e 2002 constatou que esses pacientes têm menor qualidade de vida quando comparados a crianças saudáveis e qualidade de vida igual ou maior quando comparados a grupos de crianças e adolescentes com outras doenças crônicas como artrite reumatóide juvenil, asma e epilepsia. ${ }^{26}$

\section{TRANSIÇÃO PARA AMBULATÓRIO DE ADULTOS}

Em adolescentes de 15 a 19 anos submetidos a transplante, foi constatado aumento da mortalidade em mais de três vezes quando comparados a seus pares de 10 a 14 anos. ${ }^{5,8}$ Por esse motivo, o objetivo da transição desses pacientes para o ambulatório de adultos é maximizar a longevidade com a melhor qualidade de vida possível e com o mínimo de complicações. A teoria da autodeterminação tem correlação maior com os objetivos de saúde e inclui três necessidades psicológicas: autonomia, competência e relações parentais. ${ }^{5}$

A transição para o ambulatório de adultos deve ser efetuada após terem sido atingidas algumas metas enumeradas na Figura 3.

\section{Entendimento e capacidade de descrever a causa da insuficiência hepática e a necessidade de transplante}

\section{Capacidade de saber informar sobre sua medicação}

\section{Consciência das implicações a longo e curto prazo da condição de transplantado em sua vida como um todo (prevenção de infecções, aspirações escolares, acadêmicas e vocacionais)}

\author{
4. Compreensão dos efeitos de sua doença na saúde sexual e \\ reprodutiva, incluindo: \\ - efeito da gestação no seu bem-estar \\ - efeito da medicação contraceptiva \\ - qualquer efeito teratogênico da sua medicação \\ - papel do aconselhamento genético e risco genético da sua doença \\ recorrer na prole se pertinente para sua condição
}

\author{
5. Demonstração do senso de responsabilidade na sua própria \\ condição de saúde: \\ - conhecer todos os nomes, as indicações e dosagens de sua \\ medicação \\ - conseguir solicitar renovação de suas receitas \\ - preparar sua própria medicação \\ - saber comunicar-se com seu provedor de saúde se necessário, \\ mesmo em uma situação de emergência, incluindo saber o número \\ do telefone \\ - habilidade de fazer, conservar o calendário e seguir com seus \\ cuidados
}
6. Capacidade de prover seus principais cuidados independentemente

7. Prontidão expressa de migrar para o ambulatório dos adultos

Fonte: Bell LE, Bartosh SM, Davis CL, Dobbels F, Al-Uzri A, Lotstein D, Reiss J, Dharnidharka VR on behalf of the conference attendeess. Adolescent Transition to Adult Care in Solid Organ Transplantation: a consensus conference report. Am J Transplant 2008 
Para que ocorra uma transição tranquila, os pais devem estar preparados e o paciente em boas condições de saúde. ${ }^{5}$ A equipe pediátrica precisa ser encorajada para efetuar a transferência ${ }^{33} \mathrm{e}$ a equipe de transplante de adultos precisa estar sensibilizada no sentido de efetuar as adequações ao atendimento conforme as necessidades do adolescente.

A transição poderia ser feita de várias formas, tais como: intercalando consultas nos dois ambulatórios, com o pediatra apresentando seu paciente à equipe de adultos, com um médico hebiatra nas consultas dos adolescentes nos dois ambulatórios ou um ambulatório de transição à parte do atendimento do transplante. Nossa experiência, ainda incipiente, é a presença do hebiatra nas consultas dos dois ambulatórios, permitindo ao paciente participação ativa na discussão de questões da adolescência e dos cuidados de saúde integral. Esse modelo permite uma consulta mais completa, sem que o paciente adolescente ou adulto jovem tenha necessidade de faltar a seus compromissos escolares, trabalho ou lazer.

O Departamento de Saúde do Reino Unido elaborou em 2008 um protocolo de transição ('Transition: moving on well'- from pediatric to adult health care) que facilitou o atendimento de adolescentes que necessitam de acompanhamento por doença crônica ou necessidade especial. ${ }^{34}$ Os indivíduos que sobrevivem por um longo período com expectativa sobre a natureza terminal da doença anterior ao transplante ficam despreparados para o processo de transição da infância para a adolescência e para a idade adulta. ${ }^{35}$

O pediatra, que deveria ter o papel essencial na transferência de adolescentes para serviços de saúde de adultos, muitas vezes e de modo inconsciente, acaba atuando como fator dificultador, por não demonstrar confiança plena em seus colegas. ${ }^{33}$

Infelizmente, as clínicas de adultos estão sobrecarregadas de pacientes e as questões de desenvolvimento da adolescência não são prioritárias; adolescentes com doenças crônicas podem não receber todos os cuidados de que necessitam. ${ }^{32}$

Apesar de geralmente existir uma atitude positiva em relação à transferência para o ambulatório de adultos, alguns adolescentes observaram que os profissionais de saúde do ambulatório de transição precisam melhorar o encaminhamento, principalmente no que diz respeito a informações sobre o novo local e nova equipe. ${ }^{36}$ Um estudo realizado na Suíça com 209 especialistas que atendem adultos demonstrou que $54 \%$ dos médicos não dedicavam tempo algum da consulta com a presença apenas do adolescente. ${ }^{36}$ Como a consulta transcorria integralmente com os pais, havia dificuldades na abordagem adequada de temas como sexualidade, uso e abuso tanto de álcool e tabaco como de drogas ilícitas, nutrição, questões familiares e emocionais. ${ }^{36}$ Além disso, $46 \%$ dos especialistas que atendem adultos não tiveram contato prévio com o especialista pediátrico e $58,8 \%$ não trabalhavam com o protocolo. ${ }^{36}$ No Reino Unido foi observada redução de $94 \%$ para $57 \%$ no seguimento em transplante renal após dois anos de transição. ${ }^{37}$

\section{CONSIDERAÇÕES FINAIS}

Conversar com adolescentes não é uma tarefa difícil nem toma muito tempo. O anagrama HEADS pode ser usado para lembrar o médico o que deve ser discutido: Home, Education, Activities (affect, adherence), Drugs (depression, diet) and Sex (safety, suicidality). ${ }^{14}$ As perguntas devem ser feitas de modo que não lembrem um julgamento. ${ }^{14}$

Alguns adolescentes julgam-se imortais e invulneráveis, porém nos primeiros anos da adolescência há um momento em que pensam na morte e em sua longevidade. ${ }^{14}$

Adolescência é um momento de grande desenvolvimento emocional e os adolescentes devem ter seus sentimentos respeitados, apesar de muitas vezes interpretarem erroneamente expressões faciais e a linguagem corporal dos adultos, mesmo dos profissionais de saúde do transplante. ${ }^{14}$

O desenvolvimento da identidade que ocorre na fase intermediária da adolescência poderá atrasar em pacientes transplantados, devido ao aumento da dependência aos adultos e da superproteção. ${ }^{14}$ Aqueles que se sentem bem consigo têm mais autoconfiança para formação de sua identidade, usam melhor suas habilidades e têm sensação de que são mais capazes. ${ }^{14}$ Crianças ou adolescentes com problemas psicológicos passam por mais internações hospitalares no pós-transplante hepático. ${ }^{14}$

Os pais podem sentir-se tentados a passar precocemente a responsabilidade da medicação ao filho transplantado. ${ }^{14}$ No entanto, isso deve ser feito de forma gradual, com a equipe de saúde acompanhando o processo, estipulando etapas na autonomia e encorajando-os a caprichar mais quando surgirem possíveis erros. ${ }^{14}$ Um ambulatório de transição pode facilitar uma cooperação mais estreita entre especialistas em Medicina Pediátrica e adulta, pois tais mudanças exigem que os pacientes adquiram melhor entendimento dos serviços de saúde após a transferência. ${ }^{38}$

$\mathrm{O}$ adolescente é capaz de consentir no transplante, entender sua doença, os aspectos positivos ou negativos do tratamento e os problemas de aderência da medicação, entre outros. ${ }^{14} \mathrm{O}$ jovem geralmente não gosta que seus amigos saudáveis saibam que ele está se submetendo a transplante, mas deve ser lembrado que haverá ausência das atividades escolares e seus colegas poderão imaginar que está tratando dependência de drogas ou transtorno alimentar. ${ }^{14} \mathrm{O}$ suporte melhor é dado por outros adolescentes que possa encontrar no hospital ou por grupos e associações de doenças. O período de espera para o transplante deve ser o momento de explicar sobre UTI, catéteres, dor pós-operatória e também conhecer adolescentes já transplantados.

$\mathrm{Na}$ ausência de complicações, os adolescentes recuperam-se mais rapidamente do que adultos e mais lentamente do que crianças pequenas. $^{14}$ Nessa fase, eles são mais dependentes de seus familiares e apresentam intenso vínculo com seus pais, ao contrário dos adolescentes sadios. Muitos deles têm famílias não tradicionais, onde as decisões são tomadas por avós, irmãos ou parceiro de um de seus pais. ${ }^{14}$ Por vezes, são superprotegidos e a equipe de saúde deve ser capaz de negociar gradualmente maior responsabilidade e autonomia. ${ }^{14} \mathrm{~A}$ adolescência é um período em que existe muita discordância entre pais e filhos, e a doação de um órgão por um dos genitores pode aumentar esse entrave, particularmente se os pais esperam gratidão. Também é muito difícil para os pais, especialmente para a mãe, quando ela não pode ser doadora. ${ }^{14}$

Um fator determinante para uma maior colaboração no acompanhamento do transplante é o suporte proporcionado por seus pares, que poderão ser constituídos por adolescentes da escola, da vizinhança, da igreja ou grupo de apoio do transplante ou da doença de base. ${ }^{14}$

Além da família, outro sistema de apoio muito importante 
é a escola, tanto antes como depois dos procedimentos. ${ }^{14} \mathrm{~A}$ mudança para um local próximo ao centro transplantador, associada à baixa energia no pré-transplante pode comprometer o desempenho escolar. No entanto, no pós-transplante eles ficam ansiosos por retornar à escola. ${ }^{14}$ Comorbidades, faltas à escola e transtorno de aprendizagem são complicadores do desempenho escolar. ${ }^{14}$

Um ambulatório de transição pode facilitar uma cooperação mais estreita entre pediatras e clínicos a fim de garantir aos pacientes melhor entendimento dos serviços de saúde após a transferência ${ }^{38}$ e uma oportunidade de receber orientações sobre seus cuidados de saúde, para que se torne um adulto capaz de atitudes assertivas. ${ }^{39}$ As clínicas que atendem adultos estão sobrecarregadas e as questões de desenvolvimento da adolescência infelizmente não são consideradas. ${ }^{40}$

Há muitos motivos para se comemorar o sucesso do transplante hepático: transplante de órgão sólido de melhor e maior longevidade, adaptação à vida em melhor condição de saúde e integração à sociedade, boa aceitação da imunossupressão com menos efeitos colaterais e melhor entendimento de sua ação e toxicidade. A tolerância ao enxerto existe mais como conceito do que como realidade. ${ }^{41}$ Porém, há ainda muitos degraus a se galgar na abordagem multidisciplinar do adolescente transplantado hepático e um ambulatório de transição para facilitar sua passagem para o "mundo dos adultos" é uma necessidade urgente.

\section{ABSTRACT}

Purpose: Provide information to hepatologists on the care of adolescent transplant recipients and how they can collaborate by adhering to immunosuppressive treatment to avoid risk of rejection as well as focusing other important issues of adolescence. Data source: Information was collected from scientific articles published on SciELO, MEDLINE, and PubMed databases from 2004 to 2010 , books and papers from articles of consensus of international agencies. Data synthesis: As a result of the increasing amount of transplants as well as the survival improvement, many transplanted children are attaining the turbulent adolescence period. Few transplant services have standardized a transition program to assure an adequate health's follow-up for adolescent patients after their transfer to an adult clinic. Some authors state that attention during such transitional period provides a review of education for adolescent patients as well as an opportunity to optimize the well-being in adulthood. Unfortunately, general clinics for adults are overwhelmed of patients, and the adolescent's development issues are not priorities. The pediatrician, who should be essential in transferring adolescents to adult health services, often unconsciously act as a complicating factor, since they do not show fully confident on his colleagues. Conclusion: The outpatient transition can provide opportunity to address essential issues for the overall well-being and health to adolescent liver transplant patients as well as focusing important points for their full development, including their autonomy, identity, self-esteem, quality of life, sexuality, body changes, life projects and educational / professional development.

Keywords: Adolescence, Liver Transplantation, Medication Adherence.

\section{REFERÊNCIAS:}

1. Kamath BM, Olthoff KM. Liver Transplantation in Children: Update 2010. Pediatr Clin N Am. 2010;57:401-14.

2. Mies S. Transplante de fígado. Rev Ass Med Brasil. 1998;44(2):127-34.

3. Casey BJ, Giedd JN, Thomas KM. Structural and functional brain development and its relation to cognitive development. Biol Psychol. 2000;54:241-57.

4. Herculano-Houzel S. O cérebro em transformação. Editora Objetiva 2005.

5. Bell L, Sawyer SM. Transition of care to adult services for pediatric solid-organ transplant recipients. Pediatr Clin N Am. 2010;57:593-610.

6. Knobel M, Aberastury A. La adolescência normal. Argentina, editorial Paidós, 1970.

7. Hsu, DT. Biological and psychological differences in the child and adolescent transplant recipient. Pediatr Transplantation. 2005;9:416-21.

8. Bell LE, Bartosh SM, Davis CL, Dobbels F, Al-Uzri A, Lotstein D et al. Adolescent Transition to Adult Care in Solid Organ Transplantation: a consensus conference report. Am J Transplant. 2008;8:2230-42.

9. Stam H, Hartmann EE, Deurloo JA, Groothoff J, Gootenhuis MA. Young Adult Patients with a History of Pediatric Disease: Impact on Course of Life and Transition into Adulthood. Journal of Adolescent Health. 2006;39:4-13.

10. Dommergues JP, Letierce A, Descott C, Debray D. Suivi medical, vie quotidienne et vecú de jeunes adultes après transplantation hépatique (TH) dans l'enfance. Archives de Pédiatrie. 2008;15(5):855-6.
11. Dobbels F, Van Damme-Lombaert R, Vanhaecke J, De Geest S. Growing pains: Nonadherence with the immunosuppressive regimen in adolescent transplant recipients. Pediatric Transplantation. 2005;9:381-90.

12. Berquist RK, Berquist WE, Esquive CO, Cox KL, Wayman KI, Litt IF. Adolescent non-adherence:Prevalence and consequences in liver transplant recipients. Pediatric Transplantation. 2006;10(3):304-10.

13. De Geest S, Bourgemans L, Gemoets H, Abraham I, Vlaminck H, Evers G et al. Incidence, determinants, and consequences of subclinical noncompliance with immunosuppressive therapy in renal transplant recipients. Transplantation. 1995 Feb 15;59(3):340-7.

14. Kaufman M, Shemesh E. The adolescent recipient. Pediatr Clin N Am. 2010;57:575-92.

15. RenzJF, de Roos M, Rosenthal P, Mudge C, Bacchetti P, Watson J et al. Posttransplantation Growth in Pediatric Liver Recipients. Liver Transpl. 2001;7:1040-55.

16. Bassanello M, De Palo EF, Lancerin F, Vitale A, Gatti R, Montin U et al. Growth Hormone/Insulin-Like Growth Factor 1 Axis Recovery After Liver Transplantation: A Preliminary Prospective Study. Liver Transpl. 2004;10:692-8.

17. Alonso EM. Growth and Developmental Considerations in Pediatric Liver Transplantation. Liver transplantation 2008;14:585-91.

18. Allen U, Green M. Prevention and Treatment of Infectious Complications after Solid Oran Transplantation in Children. Pediatr Clin N Am. 2010;45:459-79.

19. Sucato GS, Murray PJ. Gynecologic health care for adolescent solid organ transplant 
recipient. Pediatr Transplant. 2005 Jun;9(3):346-56.

20. Lyon M, Mc Carter R, Kuehl K. Transition to adulthood in congenital heart disease: Missed adolescent milestones. Journal of Adolescent Health. 2005 Fev;36(2):128.

21. Xia D, He HY, Liang X, Quan Y, Yan LN, Li B et al. Pregnancy after liver transplantation: Four-year follow-up of the first case in mainland China. World J Gastroenterol. 2008 December 21;14(47):7264-6.

22. Coffin CS, Shaleen AAM, Burak KW, Myers RP. Pregnancy Outcomes among Liver Transplantation recipients in the United States: A Nation Wide Case-Control Analysis. Liver Transpl. 2010 Jan;16:56-63.

23. Leithead JA, Fergusson JW, Hayes PC. Smoking-Related Morbidity and Mortality Following Liver Transplantation. Liver Transpl. 2008;14:1159-64.

24. Van der Heide F, Dijkstra G, Porte RJ, Kleibeuker JH, Haagsma EB. Smoking Behavior in Liver Transplant Recipients. Liver Transpl. 2009;15:648-55.

25. Dew MA, DiMartini AF, Steel J, Dabbs ADV, Myaskovsky L, Unruh M et al. MeaAnalysis of Risk for Relapse to Substance Use After Transplantation of the Liver or Other Solid Organs. Liver Transpl. 2007 Fev;14:159-72.

26. Anthony SJ, BarZiv SP, Ng VL. Quality of Life After Pediatric Solid Ogan Transplantation. Pediatr Clin N Am. 2007;57:559-74.

27. Laba M, Pszenny A, Dominika G, Jonas M, Durlik M, Leszsek P et al. Quality of life after liver transplantation - preliminary report. Ann Transplant. 2008;13(4):67-71.

28. Cole C, Bucuvales JC, Hornung RW, Krug S, Ryckman FC, Atherton H et al. Impact of liver transplantation on HRQOL in children less than 5 years old. Pediatr Transplant. 2004;8:222-7.

29. Anberg F, Rissanen AM, Sintonen H, Roine RP, Höckerstedt K, Isoniemi H. HealthRelated Quality of Life and Employment Status of Liver Transplant Patients. Liver Transplantation. 2009;15:64-72

30. Fredericks M, Lopez MJ, Magee JC, Shieck V, Opipari-Arrigan L. Psychological
Functioning, Nonadherence and Health Outcomes After Pediatric Liver Transplantation. American Journal of Transplantation. 2007;7:1974-83.

31. Desai R, Jamieson NV, Gimson AE, Watson J, Gibbs P, Bradley A et al. Quality of Life up to 30 years Following Liver Transplantation. Liver Transpl. 2008;14:1473-9.

32. Tong A, Morton R, Howard K et al. Adolescent experiences following organ transplantation: a systematic review of qualitative studies. J Pediatr. 2009;155(4):542-9.

33. Fox A. Physians as barriers to successfull transition care. Int J Adolesc Med Health. 2002 Jun; 9(3):364-72.

34. Pywell A. 'Transition: moving on well'--from paediatric to adult health care. British Journal of Medicine. 2008;19(10):652-6.

35. Moons P, Pinxten S, Dedroog D, Deyk K, Gewillig M, Hilderson D et al. Expectations and Experiences of Adolescents with Congenital Heart Disease on Being Transferred from Pediatric Cardiology to an Adult. Journal of Adolescent Health. 2009;44:316-22.

36. Suris JC, Akré C, Rutishauser C. How Adult Specialists Deal with the Principles of a Successful Transition. J Adolesc 2009;Dec 45(6):551-5.

37. Kipps S, Bahu T, Ong K, et al. Current methods of transfer of young people with type 1 diabetes to adults services. Diabet Med 2002;19(8):649-54.

38. Busse FP, Hiermann P, Galler A, Stumvoll M, Wiessner T, Kiess W et al. Evaluation of patients' opinion and metabolic control after transfer of young adult care. Horm Res. 2007;67(3):132-8.

39. Conway GS. Congenital adrenal hyperplasia: adolescence and transition. Horm Res. 2007;68 Suppl 5:155-7.

40. Kaufmann M. Role of adolescent development in the transition process. Prog Transpl. 2006;15(4):286-90.

41. Seyfert-Margolis V, Feng S. Tolerance: Is It Achievable in Pediatric Solid Organ Transplantation? Pediatr Clin N Am. 2010;57:523-38. 